\title{
Atom loss from Bose-Einstein condensates due to Feshbach resonance
}

\author{
V. A. Yurovsky, A. Ben-Reuven, \\ School of Chemistry, Tel Aviv University, 69978 Tel Aviv, Israel \\ P. S. Julienne and C. J. Williams \\ Atomic Physics Division, Stop 8423, National Institute of Standards and Technology, Gaithersburg, MD 20889, USA
}

(October 28, 2018)

In recent experiments on $\mathrm{Na}$ Bose-Einstein condensates [S. Inouye et al, Nature 392, 151 (1998); J. Stenger et al, Phys. Rev. Lett. 82, 2422 (1999)], large loss rates were observed when a time-varying magnetic field was used to tune a molecular Feshbach resonance state near the state of pairs of atoms belonging to the condensate many-body wavefunction. A mechanism is offered here to account for the observed losses, based on the deactivation of the resonant molecular state by interaction with a third condensate atom, with a deactivation rate coefficient of magnitude $\sim 10^{-10} \mathrm{~cm}^{3} / \mathrm{s}$.

03.75.Fi, 32.80.Pj, 32.60.+i, 34.50.Ez, 34.90.+q

Experiments have been carried out recently [1,2] (see also the review [3]) in order to control the interatomic interaction underlying the properties of a Bose-Einstein condensate (BEC). One way to achieve this 1, 2] is by tuning a magnetic field $B$ to modify the two-atom scattering length as predicted for a $B$-dependent Feshbach resonance [4. [5. The experiments carried out on a Na optical trap measured two distinct features: (a) the change in scattering length and large collisional atom losses with a slow sweep of $B$ that stopped short of the resonant field $B_{0}$, and (b) a near-catastrophic loss of atom density with a fast sweep of $B$ through the $B_{0}$ region. Two groups of investigators [6] [] have recently proposed a unimolecular mechanism to explain the latter feature as due to a fast sweep-induced transfer of population from the ground trap state of an atom pair to excited states.

We present here another possible mechanism of atom loss, which is important when the field is changed slowly, and may play a role in the fast-sweep case as well. The temporary occupation of the vibrationally-excited molecular state $m$, coupled by the resonance to atom pairs in the condensate state $a$, is converted to a stable molecular dimer state $d$ by a deactivating inelastic collision with a third atom:

$$
\begin{aligned}
& \mathrm{Na}+\mathrm{Na} \rightleftharpoons \mathrm{Na}_{2}(m) \\
& \mathrm{Na}_{2}(m)+\mathrm{Na} \rightarrow \mathrm{Na}_{2}(d)+\mathrm{Na}+\Delta E
\end{aligned}
$$

where $\Delta E$ is the excess kinetic energy released in the inelastic process. The deactivation states can be lowerlying rovibrational levels in the same spin state as the resonant level, or levels belonging to another spin state. While step (1), standing alone, is completely reversible, step (2) is irreversible, owing to the rapid ejection of the products from the condensate. The latter step usually provides sufficient kinetic energy to freely escape the trap, and is characterized by a deactivation rate coefficient $2 \gamma$ (in units of $\mathrm{cm}^{3} / \mathrm{s}$ ). The two steps described above may be followed by a third one - secondary collisions of the products with condensate atoms.

Except for $\gamma$, the magnitude of which can be extracted from fitting the experimental data, all expressions below can be derived analytically, using previously-determined parameters. A variational method was used to derive a set of three coupled Gross-Pitaevskii equations for the atomic condensate state $\varphi_{a}(\mathbf{r}, t)$, the resonant molecular state $\varphi_{m}(\mathbf{r}, t)$, and a representative deactivation state $d$. The latter can be decoupled out, leaving terms dependent on $\gamma$ in the remaining two equations (similar to those recently used by Timmermans et al. [3,8]):

$$
\begin{aligned}
i \hbar \dot{\varphi}_{a} & =\left(\frac{1}{2 m} \hat{\mathbf{p}}^{2}+V_{a}(\mathbf{r})+\mu_{a} B(t)+U_{a}\left|\varphi_{a}\right|^{2}\right) \varphi_{a} \\
& +U_{a m}\left|\varphi_{m}\right|^{2} \varphi_{a}+2 g^{*} \varphi_{a}^{*} \varphi_{m}-i \hbar \gamma\left|\varphi_{m}\right|^{2} \varphi_{a} \\
i \hbar \dot{\varphi}_{m} & =\left(\frac{1}{4 m} \hat{\mathbf{p}}^{2}+V_{m}(\mathbf{r})+\mu_{m} B(t)+U_{m}\left|\varphi_{m}\right|^{2}\right) \varphi_{m} \\
& +U_{a m}\left|\varphi_{a}\right|^{2} \varphi_{m}+g \varphi_{a}^{2}-i \hbar \gamma\left|\varphi_{a}\right|^{2} \varphi_{m}
\end{aligned}
$$

provided the deactivation state $d$ never accrues a significant population. Here $m$ is the mass of the $\mathrm{Na}$ atom; $V_{a}(\mathbf{r})$ and $V_{m}(\mathbf{r})$ are, respectively, the atomic and molecular optical trapping potentials $\left(V_{m}\right.$ including the resonance detuning for zero magnetic field); $\mathbf{r}$ is the position in the trap; $B(t)$ is the applied homogeneous magnetic field; $\mu_{a}$ and $\mu_{m}$ are, respectively, the atomic and molecular magnetic moments; and $U_{a}, U_{m}$, and $U_{a m}$ are, respectively, zero-momentum atom-atom, moleculemolecule and atom-molecule interactions, proportional to the corresponding elastic scattering lengths. The coupling constant $g$ responsible for the atom-Feshbach coupling is closely related to the parameter $\Delta$ [1.2] that characterizes the strength of the resonance shape as a function of the field B:

$$
|g|^{2}=2 \pi \hbar^{2}\left|a_{a}\right| \mu \Delta / m
$$

where $a_{a}$ is the off-resonance triplet scattering length. Calculations 6.90 give $a_{a}=3.4 \mathrm{~nm}, \mu=\mu_{m}-2 \mu_{a}=$ $3.3 \mu_{B}$ (where $\mu_{B}=9.27 \times 10^{-24} \mathrm{~J} / \mathrm{T}$ is the Bohr magneton), and $\Delta=0.001 \mathrm{mT}$ and $0.1 \mathrm{mT}$, respectively, for the two resonances observed [1.2] at $85.3 \mathrm{mT}(853 \mathrm{G})$ and 
$90.7 \mathrm{mT}(907 \mathrm{G})$. These $\Delta$ values agree with the value measured for the $90.7 \mathrm{mT}$ resonance, and with the indirectly inferred order of magnitude for the $85.3 \mathrm{mT}$ resonance.

The analysis below neglects the kinetic energy terms in Eqs. (3) and (伍), in accord with the Thomas-Fermi approximation. This reduces (3) and (4) to a system of ordinary differential equations in $\varphi_{a}(\mathbf{r}, t)$ and $\varphi_{m}(\mathbf{r}, t)$ that depend parametrically on $\mathbf{r}$. The resulting set of four real equations can then be solved numerically, for a given value of $\gamma$, starting with a Thomas-Fermi distribution or with a homogeneous (r-independent) distribution equal to the mean trap density.

We have carried out such numerical solutions for both types of MIT experiments [1,2], using the homogeneous initial conditions. In the slow-sweep experiment the timedependent magnetic field was linearly changed from an initial value of $B$ to a final value closer to resonance, and then the density was measured by shutting off the trap field and letting the condensate expand. Repeating the experiment at various final values of the magnetic field, the loss was plotted as a function of this final value. It was noted [2] that the results fit to a 3 -body rate equation for the atomic density $n(\mathbf{r}, t)=\left|\varphi_{a}(\mathbf{r}, t)\right|^{2}$, of the type $\dot{n} \approx-K_{3} n^{3}$.

Analysis of Eqs. (3, (4) shows that, out of the four real coupled equations for $\varphi_{a}$ and $\varphi_{m}$, a single rate equation for the atomic density $n(\mathbf{r}, t)=\left|\varphi_{a}(\mathbf{r}, t)\right|^{2}$ can be extracted, if the following condition (called here the "fast decay" approximation)

$$
|g|^{2} \ll \hbar^{2} \gamma^{2} n(\mathbf{r}, t)
$$

(i.e., $\left.n_{m}(\mathbf{r}, t)=\left|\varphi_{m}(\mathbf{r}, t)\right|^{2} \ll n(\mathbf{r}, t)\right)$ is observed. The resulting rate equation attains the nonlinear form $\dot{n}(\mathbf{r}, t)=-F(n(\mathbf{r}, t), \mathbf{r}, t)$, where

$$
F(n, \mathbf{r}, t)=\frac{6|g|^{2} \gamma n^{3}}{[V(\mathbf{r})-\mu B(t)]^{2}+(\hbar \gamma n)^{2}}
$$

and $V(\mathbf{r})=2 V_{a}(\mathbf{r})-V_{m}(\mathbf{r})$. Eq. (7) can be expressed in terms of a collision cross section of the Breit-Wigner shape (dependent on the position $\mathbf{r}$ in the trap) for zeromomentum collisions, in which $2 \gamma n$ is the width of the output channel, and the width of the input channel is proportional to $|g|^{2}$. This observation establishes a link between the macroscopic approach used here and microscopic approaches, treating the loss rate as a collision process.

It follows from Eq. (7), that very close to resonance (where $B(t)$ is within $1 \mu \mathrm{T}$ of a resonance), the behavior of Eq. (7) changes its form gradually from that of a 3 -body to that of an effective 1-body rate equation. However, all values of $B$ used for the slow-sweep experiments of the MIT group lie outside this narrow region, where the "off-resonance" condition

$$
\hbar \gamma n(\mathbf{r}, t) \ll|V(\mathbf{r})-\mu B|
$$

holds. Given this condition, one can write Eq. (7) (to a very good approximation) in the 3-body form $F(n, \mathbf{r}, t)=K_{3}(\mathbf{r}, t) n^{3}$ with

$$
K_{3}(\mathbf{r}, t)=\frac{12 \pi \hbar^{2}\left|a_{a}\right| \gamma \Delta}{m \mu\left[B(t)-B_{0}(\mathbf{r})\right]^{2}}
$$

where $B_{0}(\mathbf{r})$ is the local resonance value of $B$. We shall refer to the combination of Eqs. (6) and (8), leading to Eq. (9), as the "3-body" approximation. This expression is similar to the one derived earlier [3, 8] up to a factor $3 / 2$, which reflects the fact that 3 atoms are lost per one deactivating collision.

For $K_{3}$ given by Eq. (9), the rate equation may be solved analytically in the form

$$
\begin{aligned}
n(\mathbf{r}, t)=n\left(\mathbf{r}, t_{0}\right) & {\left[1+24 \pi \hbar^{2}\left|a_{a}\right| \Delta \gamma n^{2}\left(\mathbf{r}, t_{0}\right)\right.} \\
\times & \left.\left(t-t_{0}\right) /\left(m \mu \dot{B}^{2} t t_{0}\right)\right]^{-1 / 2}
\end{aligned}
$$

where $\dot{B}$ is the magnetic-field ramp speed, and the starting time $t_{0}$ is measured from the extrapolated resonancecrossing time as $t=0$. The graphs shown in Figs. 1 and 2 pertain to the slow-sweep experiment conducted at MIT, and more particularly to the strong $90.7 \mathrm{mT}$ resonance. This resonance has been approached from below with two ramp speeds, and from above - with one. The difference between Eqs. (9, 10) and the results of a direct numerical solution of Eqs. (3) and (4) for all ramp speeds in the $K_{3}$ plots (Fig. 1) is so small, that the corresponding plots are indistinguishable. The remaining atomic density, at the moment $t$ of stopping the ramp (see Fig. 2), was also calculated, using the homogeneous-density initial condition, starting from a $B$ value of $89.4 \mathrm{mT}$ on approach from below and $91.6 \mathrm{mT}$ from above, for which the initial mean densities were extracted from the experimental data [2. The graphs clearly show a best fit with $\gamma$ of the order of $10^{-10} \mathrm{~cm}^{3} / \mathrm{s}$, which (given a density of about $10^{15} \mathrm{~cm}^{-3}$ ) implies a deactivation time of $\sim 10^{-5} \mathrm{~s}$.

The magnitude of the inelastic rate coefficient $2 \gamma$ needed to explain the data is quite plausible. It is two orders of magnitude smaller than the upper limit allowed by the unitarity constraint on the $S$-matrix. According to this condition [10], in the limit of small momentum, $2 \gamma \leq \hbar \lambda / m$, where $\lambda$ (the de Broglie wavelength) is in turn limited by the trap dimensions. This requirement puts an upper bound of $2.5 \times 10^{-8} \mathrm{~cm}^{3} / \mathrm{s}$ to $2 \gamma$ in the case considered here. Furthermore, our estimate of $10^{-10} \mathrm{~cm}^{3} / \mathrm{s}$ for $\gamma$ is consistent with calculations carried out recently [11] on the rate of vibrational deactivation in $\mathrm{H}_{2}+\mathrm{He}$ ultracold collisions, producing a comparable result in spite of the large energy gaps in $\mathrm{H}_{2}$.

It is interesting to compare the outcome of the deactivation mechanism described here with that of other loss mechanisms based on a unimolecular process (not involving a third atom) [6.7], proposed for the second type of 
MIT experiment with the fast sweep of B through resonance. To see this one can remove the imaginary term in Eq. (3), and replace $\gamma n$ in Eq. (4) with an $n$-independent $\Gamma$. In the fast-decay limit, in which $n_{m} \ll n$, both mechanisms would yield similar results for $K_{3}$ and $n$ of the slow-sweep experiments, if similar decay times $\left(\sim 10^{-5} \mathrm{~s}\right)$ were to be reckoned in both processes.

The 3-body approximation does not hold very close to resonance, and is therefore inapplicable to a description of the fast-sweep experiment, in which the Zeeman shift was swept rapidly through the resonance, causing the most dramatic loss rates (see Refs. [1,2]). Nevertheless, maintaining only the fast decay approximation, Eq. (6), a simple analytical expression can be obtained also for this kind of measurement. Assuming the magnetic field variation is long enough to allow taking the asymptotic time limits, reached under the condition $\mu \delta B \gg \hbar \gamma n$ where $\delta B$ is the range of variation of $B$ on either side of the resonance, the ramp starting and stopping times can be extended to $\pm \infty$. One then obtains at all positions $\mathbf{r}$

$$
n(\mathbf{r}, \infty)=\frac{n(\mathbf{r},-\infty)}{1+s n(\mathbf{r},-\infty)}, \quad s=\frac{12 \pi^{2} \hbar\left|a_{a}\right|}{m} \frac{\Delta}{|\dot{B}|} .
$$

This asymptotic result (as opposed to the one mentioned earlier with regard to the slow-sweep MIT experiment) is independent of $\gamma$.

Assuming, as before, a homogeneous initial density within the trap, Eq. (11) also describes the loss of the total population $N(t)=\int n(\mathbf{r}, t) d^{3} r$. An analytical asymptotic expression for the total population can also be found when the homogeneous distribution is replaced by the Thomas-Fermi one (given in Ref. [12]), in which case

$$
\begin{aligned}
& \quad \frac{N(\infty)}{N_{0}(-\infty)}=\frac{15}{2 s n_{0}}\left[\frac{1}{3}+\frac{1}{s n_{0}}-\frac{1}{2 s n_{0}} \sqrt{1+\frac{1}{s n_{0}}}\right. \\
& \left.\times \ln \left(\left(\sqrt{1+\frac{1}{s n_{0}}}+1\right) /\left(\sqrt{1+\frac{1}{s n_{0}}}-1\right)\right)\right]
\end{aligned}
$$

where $n_{0}$ is the maximum initial density in the center of the trap.

The analytical results of Eq. (11), together with the direct numerical solutions of Eqs. (3) and (4), are compared in Fig. 4 with the results of the fast-sweep experiment [1,2]. The numerical results show that if the deactivation time is not small enough compared to the sweep time, and the magnetic field variation is not large enough, the assumptions underlying Eqs. (11) and (12) do not hold, and thus the condensate loss will depend on $\gamma$. These calculations show a rough agreement with the measured loss rates for values of $\gamma$ of the orders of $10^{-9}-10^{-11} \mathrm{~cm}^{3} / \mathrm{s}$, though these results are not as sensitive to $\gamma$ as were the fits to the slow-sweep experiments discussed above.

The deactivation reaction Eq. (2) produces $4 \gamma n n_{m}$ "hot" particles (atoms and molecules) in a unit volume per unit time. Traversing a distance $b$ at a speed $v$, these particles create new hot particles in a cascading process. The density of hot particles may then be estimated as $n_{h} \approx 4 \gamma n n_{m} b / v \exp \left(b \sigma\left(n+n_{m}\right)\right)$, where $\sigma$ is the elastic collision cross-section, considered here identical for atom-atom and atom-molecule collisions. The rate of additional loss due to these secondary collisions is estimated as $v \sigma n n_{h}$ for the atomic condensate and $v \sigma n_{m} n_{h}$ for the molecular condensate. This loss rate depends essentially on the product $\sigma b$. A conservative estimate of this product is obtained by taking $\sigma=0.5 \times 10^{-12} \mathrm{~cm}^{2}$ (the elastic Na-Na cross section at $0.1 \mathrm{~K}$ [13], a typical vibrational deactivation energy), and $b=10^{-3} \mathrm{~cm}$ (the condensate-cloud radius). A more realistic value of this product may be higher on account of the larger axial condensate length, the rise of the elastic cross section at energies below $\sim 10 \mathrm{mK}$ 13], and a possible contribution of inelastic collisions. Two values of $\sigma b$ are therefore used in Fig. 4. This figure shows that the effect of secondary collisions, calculated for a homogeneous density, may appreciably increase the condensate loss, while the use of a Thomas-Fermi density [see Eq. (12)] should decrease it.

The authors are most grateful to Wolfgang Ketterle and Joern Stenger for helpful information regarding the MIT experiments.

[1] S. Inouye, M. R. Andrews, J. Stenger, H.-J. Miesner, D. M. Stamper-Kurn, and W. Ketterle, Nature 392, 151 (1998).

[2] J. Stenger, S. Inouye, M. R. Andrews, H.-J. Miesner, D. M. Stamper-Kurn, and W. Ketterle, Phys. Rev. Lett. 82, 2422 (1999).

[3] E. Timmermans, P. Tommasini,M. Hussein, and A. Kerman, Phys. Rep., to be published (1999).

[4] E. Tiesinga, B.J. Verhaar, and H.T.C. Stoof,Phys. Rev. A47, 4114 (1993); E. Tiesinga, A.J. Moerdijk, B.J. Verhaar, and H.T.C. Stoof, Phys. Rev. A46, R1167 (1992).

[5] A. J. Moerdijk, B. J. Verhaar, and A. Axelsson, Phys. Rev. A 51, 4852 (1995).

[6] F. H. Mies, P. S. Julienne, and E. Tiesinga, prepublication manuscript (1999).

[7] F. A. van Abeelen, and B. J. Verhaar, "Giant losses in a recent Feshbach resonance scattering experiment at MIT", Workshop on Formation of Cold Molecules, 1 - 5 March 1999, Les Houches, France (unpublished).

[8] E. Timmermans, P. Tommasini, R. Côté, M. Hussein, and A. Kerman, Phys. Rev. Lett. (submitted); condmat/9805323.

[9] F. A. van Abeelen, and B. J. Verhaar, as discussed in Ref. [2].

[10] P. S. Julienne and F. H. Mies, J. Opt. Soc. Am. B 6, 2257 (1989); P. S. Julienne, A. M. Smith, and K. Burnett, Adv. At. Mol. Opt. Phys. 30, 141 (1993).

[11] R. C. Forrey, V. Kharchenko, N. Balakrishnan and A. Dalgarno, Phys. Rev. A 59, 2146 (1999).

[12] M.-O. Mewes, M. R. Andrews, N. J. van Druten, D. M. 
Kurn, D. S. Durfee, and W. Ketterle, Phys. Rev. Lett. 77, 416 (1996).

[13] R. Côté, and A. Dalgarno, Phys. Rev. A50, 4827 (1994).

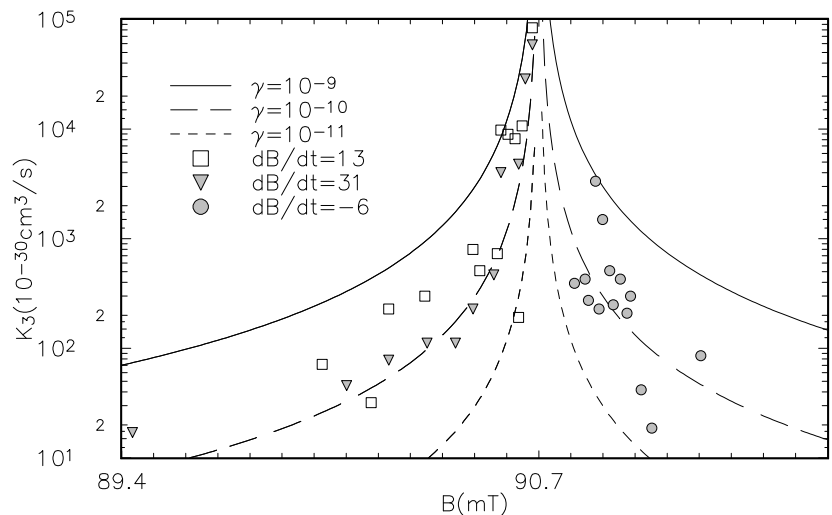

FIG. 1. The 3-body rate coefficient $\left(K_{3}\right)$ vs. the stopping value of the magnetic field, calculated with 3 different values of the deactivation rate $\gamma$ (in units of $\mathrm{cm}^{3} / \mathrm{s}$ ), on approaching the resonance from below or above. These are compared with the experimental results (Ref. [2]). Ramp speeds shown are in units of $\mathrm{mT} / \mathrm{s}$.

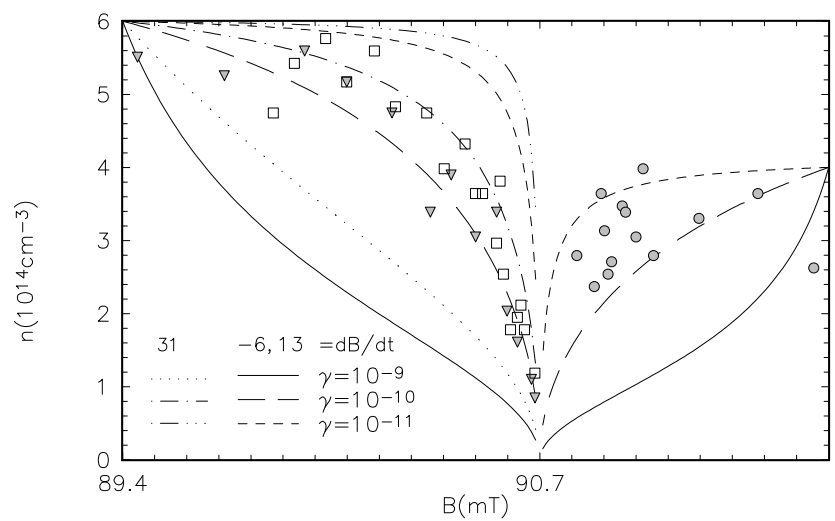

FIG. 2. The surviving mean density vs. the stopping value of the magnetic field, calculated with 3 different values of the ramp speed $\dot{B}$ (in $\mathrm{mT} / \mathrm{s}$ ). Other notations as in Fig. 1 .

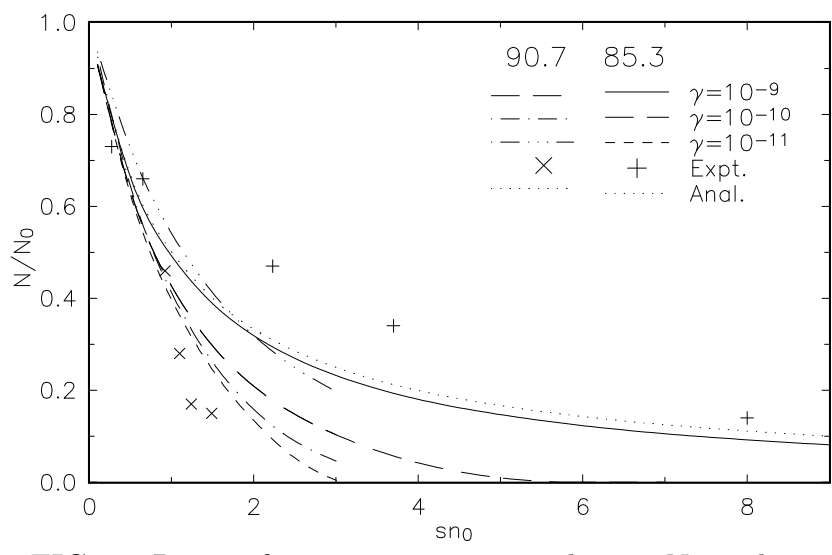

FIG. 3. Ratio of surviving trap population $N$ to the initial one $N_{0}$ for the $85.3 \mathrm{mT}(853 \mathrm{G})$ and $90.7 \mathrm{mT}$ (907G) resonances in the homogeneous-density approximation vs. $s n_{0}$ (where the parameter $s$ is defined by Eq. (11) and $n_{0}$ is the initial density). The curves show calculations carried out for different magnitudes of the coefficient $\gamma$ (in units of $\mathrm{cm}^{3} / \mathrm{s}$ ), and with the analytical result of the fast-decay approximation Eq. (11) (dots). The plots for the $90.7 \mathrm{mT}$ resonance with $\gamma=10^{-9} \mathrm{~cm}^{3} / \mathrm{s}$ and for the $85.3 \mathrm{mT}$ resonance with $\gamma=10^{-10} \mathrm{~cm}^{3} / \mathrm{s}$ are practically indistinguishable. The calculations are compared with the results of the MIT fast-sweep experiment (Ref. 2]).

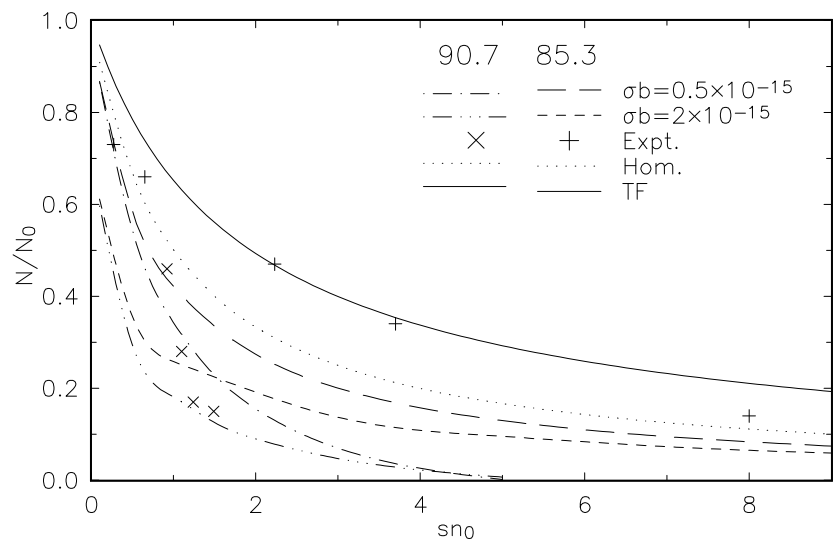

FIG. 4. Same as Fig. 3, but taking account of secondary collisions for $\gamma=10^{-9} \mathrm{~cm}^{3} / \mathrm{s}$ and various values of the parameter $\sigma b$ (in units of $\mathrm{cm}^{3}$ ). The analytical results (without taking account of secondary collisions) for the Thomas-Fermi distribution Eq. (12) (solid line) and the homogeneous distribution Eq. (11) (dots) are plotted as well. 